Luoghi medii delle stelle di confronto.

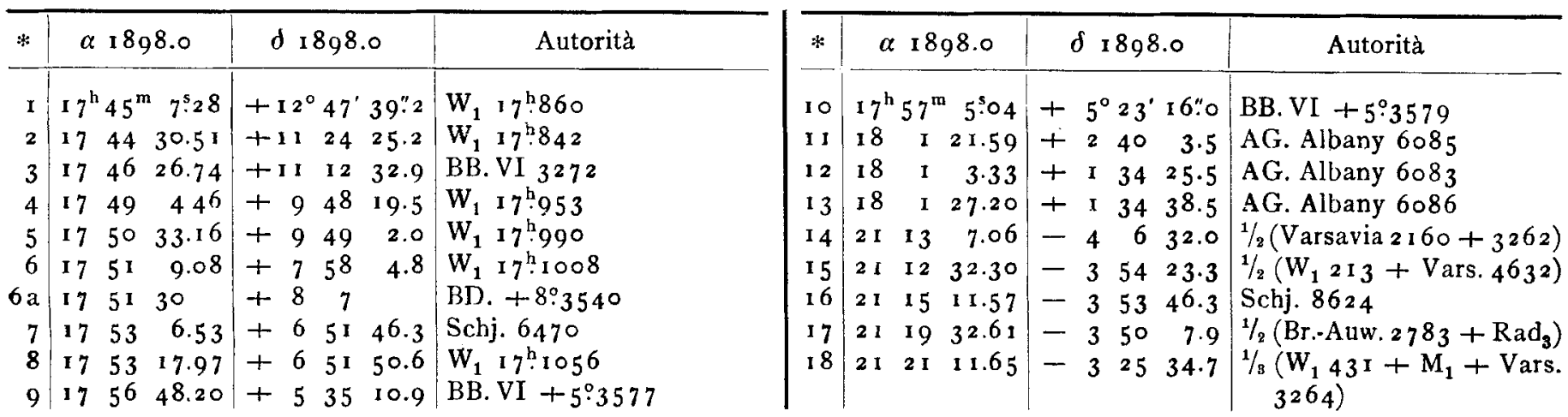

* 7. Sj. $-\mathrm{W}_{1}$ 1049 $=-0 . \mathrm{s} 4$-16"8. Questa * 7 ha in Weisse una declinazione maggiore del dovere di $10^{\prime \prime}$ per errore di riduzione dalla Zona 93 di Bessel, ciò che si fa subito manifesto paragonando le differenze di declinazione fra $* 7 \mathrm{e} * 8$ date dalla Zona e quelle date dal Weisse.

$$
\begin{aligned}
& \text { Le osservazioni di Bessel danno: } * 8-* 7=\begin{aligned}
& +11^{5} \cdot 30-3 " 7 \\
& +11.54+6.6,
\end{aligned} \\
& \text { Le mie }
\end{aligned}
$$

cioè io trovai la $* 8$ seguente più boreale press' a poco come è indicato nella nota a pag. 238 del catalogo di Schjellerup. Framezzo a queste due stelle ve n'è un' altra (pure notata da Schjellerup) grande come * 7 seguente 4.4 più boreale $47^{\prime \prime}$ la quale verrebbe ad occupare nella BD. il posto $+6: 35^{82}$ bis.

Arcetri-Firenze, 1898 Nov. 30 .

\begin{tabular}{|c|c|c|c|c|c|c|c|c|c|c|}
\hline 1898 & T.m.Arcetri & $\Delta \alpha$ & $\Delta \delta$ & Cf. & $\alpha$ app. & $\log p .4 \mid$ & $\delta$ app. & $|\log p .4|$ & Red. ad l. app. & $*$ \\
\hline
\end{tabular}

A. Abetti.

Osservazioni di asteroidi ad Arcetri.

Pianeta $1898 \mathrm{DU}$.

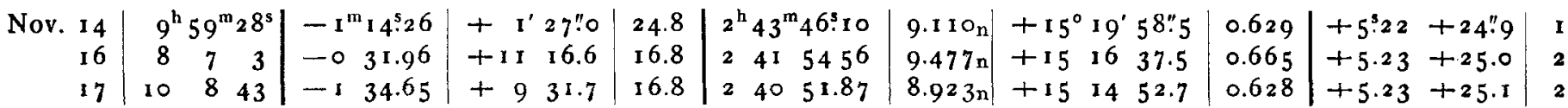

Grandezza 12.5 .

Pianeta 1898 ED. (Continuazione, v. No. 3534 ).

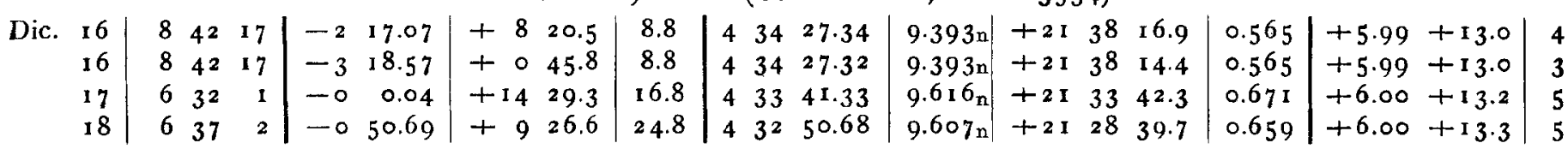

\begin{tabular}{|c|c|c|c|}
\hline 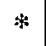 & $\alpha \quad 1898.0$ & $\delta 1898.0$ & Autorità \\
\hline $\mathbf{I}$ & $2^{\mathrm{h}} 44^{\mathrm{m}} 55^{5} .14$ & $+15^{\circ} 18^{\circ} 6^{\prime \prime} 6$ & AG. Berlin A. 767 \\
\hline 2 & $242 \quad 21.29$ & +15455.9 & AG. Berlin A. 755 \\
\hline 3 & $\begin{array}{lll}437 & 39.90\end{array}$ & +213715.6 & BB.VI $+21.69 I$ \\
\hline
\end{tabular}

Grandezza II.5.

Luoghi medii delle stelle di confronto.

\begin{tabular}{|c|c|c|c|}
\hline$*$ & $\alpha \quad 1898.0$ & $\delta 1898.0$ & Autorità \\
\hline 4 & $4^{h} 3^{6^{m}} 3^{8 s} \cdot 4^{2}$ & $+21^{\circ} 29^{\prime} 43^{\prime \prime} 4$ & BB. VI +21 9687 \\
\hline 5 & $\begin{array}{lll}4 & 33 & 35.37\end{array}$ & $\begin{array}{lll}+21 & 18 & 59.8\end{array}$ & AG. Berlin B. 1482 \\
\hline
\end{tabular}

Arcetri-Firenze, 1898 Dic. 19.
A. Abetti.

\title{
Beobachtung des Planeten (433) Eros
}

am 14 zöll. Equatoréal coudé der k. k. Sternwarte zu Wien von Dr. F. Bidschof.

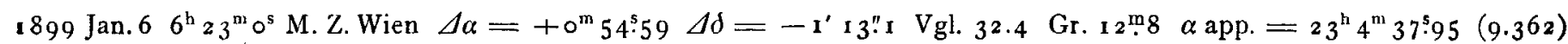
$\delta$ app. $=+4^{\circ} 4^{\prime} 19^{\prime \prime} 9(0.788)$ Red. ad l, app. +0.46 +6." .

Vergleichstern (1899.0): $\quad \alpha=23^{\mathrm{h}} 3^{\mathrm{m}} 42^{\mathrm{s}} .90 \quad \dot{\delta}=+4^{\circ} 47^{\prime} 26^{\prime \prime} .9$ AG. Albany 7987 . 